\title{
Cost-Effective Time-Redundancy based Optimal Task Allocation for the Edge-Hub-Cloud Systems
}

\author{
Andreas Kouloumpris, Theocharis Theocharides and Maria K. Michael \\ KIOS Research and Innovation Center of Excellence \\ Department of Electrical Engineering, University of Cyprus \\ Nicosia, Cyprus \\ \{kouloumpris.andreas, ttheocharides, mmichael\}@ucy.ac.cy
}

\begin{abstract}
Shifting tasks from the cloud to the edge Internet of Things (IoT) devices facilitates real-time decision support, yielding rise to the emerging edge-hub-cloud computing architecture. However, as edge devices tend to be more vulnerable to errors due to environmental/technological factors, the reliability of the application can be compromised due to this shift. We propose an optimization and design exploration framework for task allocation which maximizes reliability while respecting latency and energy constraints in computation and communication tasks, as well as memory computation constraints. It considers varying tasks' vulnerability factors, per possible executing device, and allows for different task re-execution reliability approaches based on full or selective dual or triple re-execution of tasks. We validate and showcase the effectiveness of our approach through a real-life case study for power tower/line inspection using UAVs (Unmanned Aerial Vehicles). Evaluation results show that our framework is capable of optimizing the overall reliability of the targeted application while satisfying strict latency, energy, and memory constraints, allowing the designer to explore different reliability approaches in a short period of time.
\end{abstract}

\section{INTRODUCTION}

In recent years, facilitated by the need for real-time decision support and near-sensor processing, computation has started to move from the cloud, towards the hub and the edge [1]. More specifically, an increasing shift towards the edge IoT devices has become a trend, especially for performance-oriented and mission-critical applications, such as search and rescue or critical infrastructure monitoring, yielding rise to the so-called edgehub-cloud computing paradigm [1]. Through this paradigm, the edge and hub devices along with the cloud infrastructure collaborate to collectively handle emerging application demands.

Exchanging and processing data through the cloud can be considered more reliable, in contrast to edge devices that are typically resource-constrained and demand low power. Moreover, due to reduced cost requirements, edge and possibly hub devices, are typically more vulnerable than server infrastructure to various reliability threats caused by technology scaling and large integration [2] (such as wear-out and aging failure mechanisms) as well as environmentally induced errors (such as soft-errors). This occurs as costly error detection and correction mechanisms are more affordable at the high-end devices than the low-end edge devices.

As it is a well-known fact that task vulnerability factors are workload and device-dependent [3], task allocation plays an

This work is funded by the European Union's Horizon 2020 research and innovation programme under grant agreement No 739551 (KIOS CoE) and from the Republic of Cyprus through the Directorate General for European Programmes, Coordination and Development. important role in the design flow, in the sense that different allocations result in different overall system reliability. Therefore, the problem of task allocation emerges as a primary challenge, in terms of which tasks should be executed on the edge, hub, and the cloud infrastructure, to optimize system reliability.

Further to reliability-aware task allocation, the designer can use either time redundancy or hardware redundancy approaches to further improve reliability. Hardware redundancy is often a preferred choice because it can offer high reliability and performance, especially when involving safety-critical applications for which real-time response is a requirement. However, IoT device design relies on not only low-power consumption but also on low manufacturing cost. This implies that hardware redundancy may not be the best choice since it can incur notable overheads, thus an increased overall cost. Consequently, a suitable approach to increase reliability is time redundancy, as long as, in real-time systems, the overall application latency adheres to its deadline constraints.

This work considers the edge-hub-cloud system (i.e., a single edge device, a single hub device, and the cloud) and time redundancy approaches based on task re-execution and proposes a cost-effective task allocation framework capable of considering various task characteristics and constraints such as devicedependent task's vulnerability factor, task's computation and communication latency, energy constraints, as well as task's memory requirements. In this work we consider ideal communication channel operation. The proposed optimization framework delivers optimal task allocation that maximizes the reliability of the system. In particular:

- The proposed framework enables the designer to integrate methods such as Dual Re-execution (DR), Triple Re-execution (TR), as well as a Hybrid Re-execution (HR) approach (a mixture of DR and TR). All approaches can be applied fully (i.e., all tasks use the same approach) or selectively, by choosing which tasks to be executed once, twice, or three times based on their respective vulnerability factors.

- The framework enables cost-effective design exploration, permitting the designer to investigate various levels of time redundancy, by adjusting the tolerable vulnerability threshold. This threshold essentially defines different task criticality levels concerning reliability, enabling the designer to define which tasks require DR or TR, based on their pre-computed architectural vulnerability factors [4]. For example, if the tolerable user-defined vulnerability threshold is $X(Y)$ for DR $(T R)$, a task with a vulnerability factor greater than the DR 
(TR) threshold will be executed twice (three times).

- The proposed framework takes into consideration several application-specific constraints and parameters that can be computed via profiling, such as task's computation latency, task's energy consumption, and task's primary and secondary memory footprints. The communication latency and energy consumption for each task between the edge and the hub, as well as the hub and the cloud, are also considered.

- We demonstrate our framework through a real-world case study while considering several scenarios, that is, various combinations between edge and hub devices and using different bandwidth values for each communication channel, to provide a qualitative and quantitative evaluation of the proposed framework and illustrate its usage capabilities.

In the remaining of this paper, we introduce preliminaries and related work in Section II. The proposed optimization framework is presented in Section III. Section IV provides an evaluation of the proposed framework using a real-life case study involving critical infrastructure monitoring. We conclude the paper in Section V.

\section{Preliminaries \& Related Work}

While edge computing is a broad term that covers devices from servers to sensors, in this work we focus on the paradigm described earlier, such as remotely deployed devices and sensors, controlled through an equally mobile computing hub, that subsequently communicates with the cloud infrastructure. Such edge devices, in particular, are characterized by limited computational capabilities, restricted by energy and memory constraints, and often operate in harsh environments with increasing reliability requirements. Within the edge-hub-cloud computing paradigm, therefore, the challenge is to identify which tasks should be allocated at the edge, the hub, and the cloud, respectively, such that design metrics are satisfied [5]. Task allocation has been an ongoing challenge in several paradigms ([6], [7]), in both homogeneous and heterogeneous systems. However, only recently it has received attention within the context of edge computing (as described above), especially as emerging applications that require real-time decision support migrate towards the edge of the infrastructure. Trying to solve the problem, relevant research focuses on minimizing the overall latency of the system, subject to other constraints such as energy consumption, resources, etc.

Within this context, [8] proposes a two-step decision process for choosing which tasks will be executed locally at the IoT nodes where they are present while the rest will be migrated to a group of peer nodes in the network or on a fog/cloud server, to maximize performance. In [9], the authors propose a combined fog/cloud architecture to minimize the overall latency of the services that request resources by reducing the cloud access delay in an IoT situation. In a similar attempt, [10] proposes a formulation of a computation offloading game, to allocate fog computing resources to the IoT users in a hierarchical computing paradigm (fog/cloud), such that, each user is maximizing its quality of experience. [11] proposes a framework capable of producing the optimal task allocation within the edge-hub-cloud computing paradigm, in such a way that several operational and

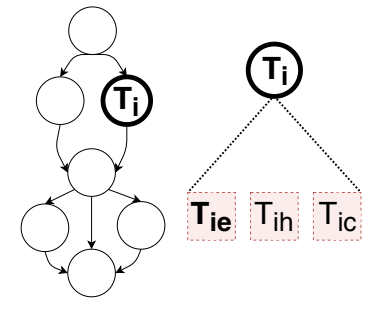

(a) (b)

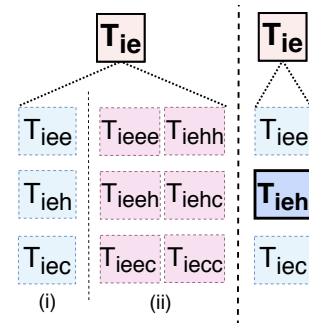

(c)

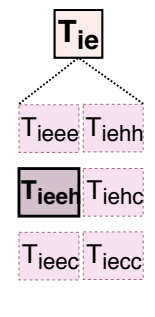

(e)
Fig. 1: Graph transformation of the traditional task flow graph for each $T_{i}$ : (a) a generic task flow graph; (b) the edge-hub-cloud (EHC) version of $T_{i}$; (c) reliability-aware EHC version of $T_{i e}$ with (i) DR options (ii) TR options; (d) $T_{i}$ will be executed twice, once on the edge and once on the hub device; (e) $T_{i}$ will be executed three times, twice on the edge device and once on the hub device

resource constraints are satisfied while the overall latency of the system is minimized.

While all aforementioned works focus exclusively on performance (minimizing the latency), Hou et al. [12] introduced a fog-computing based computation offloading into a swarm of drones instead of the cloud-based one, targeting minimal energy consumption, subject to latency and reliability constraints. Related works in [13] and [14] explore the problem of task allocation targeting reliability improvement ([13]) and joint latency-reliability trade-offs exploration ([14]) within the context of network infrastructure, focusing on servers at the edge of the network (i.e. edge servers) and mobile computing devices such as laptops, tablets, and mobile phones. The work in [15] proposes the integration of reliability techniques and specifically using dual redundancy within the task allocation problem while still targeting minimum latency. In [16], the authors propose budgeting of the introduced effective critical bits, a metric that captures the application's reliability impacting factors, to enable the system to select the proper fault tolerance techniques, thus, maximizing the performance while satisfying the reliability demands of the application in reconfigurable architectures.

In contrast to all previous works discussed above, this work addresses the problem of task allocation within the edge-hubcloud computing paradigm such that the overall reliability of the system is maximized while considering latency, energy, and memory constraints. Moreover, the proposed optimization framework can integrate various reliability improvement mechanisms based on DR and TR.

\section{OPTIMIZATION AND DESIGN-EXPLORATION FRAMEWORK}

\section{A. Task Graph Transformation}

The proposed framework receives as an input an application task flow graph (TFG). We utilize a similar approach as in [11], which transforms the task flow graph into an edge-hub-cloud graph (EHCG). Each task node in the TFG is replicated up to three different tasks nodes in the EHCG, referred to as candidate task nodes, that is, $\forall T_{i} \in \mathrm{TFG} \exists\left\{T_{i e}, T_{i h}, T_{i c}\right\}$ (see Fig. 1(b)). For each of the three candidate task nodes in the EHCG only one will be allocated for execution. Hence, each task $T_{i}$ in the original TFG, can be executed either on the edge $(e)$ device, the hub $(h)$ device, or the cloud $(c)$ unit. 
To include re-execution mechanisms such as DR and TR, the EHCG is further transformed into its reliability-aware version, as shown in Fig. 1(c). Each task in the EHCG can be transformed into its DR version such that $\forall T_{i j} \in$ EHCG $\exists\left\{T_{i j k} \mid j, k \in\{e, h, c\}\right\}$ or its TR version such that $\forall T_{i j} \in$ EHCG $\exists\left\{T_{i j k l} \mid j, k, l \in\{e, h, c\}\right\}$. When $T_{i j}$ is transformed into its DR counterpart, i.e. $T_{i j k}$, the $T_{i}$ executed at unit $k$, will send its computation results back to $T_{i j}$ where a comparison will take place. For example, as shown in Fig. 1(d), $T_{i}$ is allocated to be executed twice, once on the edge and once on the hub device and then the comparison will take place on the edge. Similarly, when $T_{i j}$ is transformed into its TR counterpart, $T_{i j k l}$, the selected $T_{i}$ executed at the unit $k$ and unit $l$, will send their computation results back to $T_{i j}$ where the voting procedure will take place. For instance, as shown in Fig. 1(e), $T_{i}$ will be executed three times, twice on the edge device and once on the hub device, where the voting will take place at the edge device. To encode these re-execution mechanisms selectively, user-defined DR $\left(\mathcal{T}_{\mathcal{D} \mathcal{R}}\right)$ and $\mathrm{TR}\left(\mathcal{T}_{\mathcal{T} \mathcal{R}}\right)$ tolerable vulnerability thresholds are considered, where $\mathcal{T}_{\mathcal{T} \mathcal{R}}>\mathcal{T}_{\mathcal{D} \mathcal{R}}$, to determine whether a task in the TFG is transformed into its DR version or its TR version. For example, if task $T_{i e}$ (to be executed at the edge), has a vulnerability factor greater than $\mathcal{T}_{\mathcal{D} \mathcal{R}}$ but less than $\mathcal{T}_{\mathcal{T} \mathcal{R}}$, then it is transformed into 3 candidate tasks, one for each DR scenario (see Fig. 1(c)(i)). Similarly, if $T_{i e}$ has a vulnerability factor greater than the $\mathcal{T}_{\mathcal{T} \mathcal{R}}$, then it can be transformed into six candidate tasks, one for each possible TR scenario with the first execution at the edge and the remaining two at any device (see Fig. 1(c)(ii)). It must be emphasized that our framework explores all possible combinations/options of DR and TR among the edge-hub-cloud devices.

Each task node in the reliability-aware EHCG (R-EHCG) represents a candidate task along with its parameters, such as vulnerability factor, execution time, main and secondary memory usage, and energy consumption. Each edge in the REHCG shows the precedence relationship between the candidate tasks. In particular, each edge incorporates the communication latency and communication energy needed to exchange the necessary information between the two candidate tasks. We assume that the edge and the cloud units do not communicate directly, but instead, their communication is established through the hub device. We use a similar energy model as the one used in [17], in which the energy consumed by each candidate task is the average power consumption of the specific device running the specific application task times its execution time. Due to space limitation we refer the readers in [17] for more details.

\section{B. Reliability Optimization Problem Formulation}

In general, the reliability of a system $\left(R_{s}\right)$ with components in series is given by: $R_{s}=\prod_{i=1}^{n}\left(R_{i}\right)$, where $R_{i}$ is the reliability of the $i_{t h}$ component. To compute the reliability of the candidate tasks which encapsulate dual or triple re-execution mechanisms, we use the formula which concerns a system with components in parallel. Therefore, we calculate the probability of a system with components in parallel to fail $\left(F_{s}\right)$, which is given by: $F_{s}=\prod_{i=1}^{n}\left(1-R_{i}\right)$. It should be noted that in this work, $F_{i}$, the probability of the $i_{t h}$ component to fail, is also denoted as the task vulnerability factor $V_{i}=1-R_{i}$. Since these metrics are probabilistic, that is, we can not simply add their values, one major contribution of this paper is that we ensure that the formulation will remain linear for complexity purposes, by keeping the objective function in the form of a simple summation. This is achieved by transforming the reliability value of each candidate task into its logarithmic form. This holds since the smallest (greatest) reliability values will be represented by the smallest (greatest) logarithmic values. Given that, $\log \left(\prod x_{i}\right)=\sum \log \left(x_{i}\right)$ and since our system consists of components in series, which in our case are the candidate tasks, the overall reliability of the system is the product of the reliability of the individual candidate task given by: $\log \left(\prod_{i=1}^{n}\left(R_{i}\right)\right)=\sum_{i}^{n} \log \left(R_{i}\right)$, where $R_{i}=1-V_{i}$

1) Variables and Parameters: To formulate the problem, we consider the R-EHCG and we define two binary decision variables $x_{i j}$ and $x_{i j k}$ per each node and edge in the graph, respectively. The variable $x_{i j}$ is set to 1 when task $i\left(T_{i}\right)$ is allocated to be executed on computational unit $j\left(T_{i j}\right)$, and variable $x_{i j k}$ is set to 1 when the outgoing link $j \rightarrow k$ of $T_{i}$ is activated. In addition, let $R_{i j}, E_{i j}, M_{i j}, S_{i j}$ and $L_{i j}$, be the reliability value, energy consumption, main and secondary memory usage, and the execution time of $T_{i j}$, respectively. Moreover, let $C_{L_{i j k}}$ and $C_{E_{i j k}}$ be the communication latency and communication energy for $T_{i}$ to send a specific amount of data from computational unit $j$ to computational unit $k$. Finally, let the $E_{j}, M_{j}, S_{j}$ and $L_{\text {thresh }}$ be the designer defined energy and memory budgets for each computational unit and the threshold for the overall latency of the system.

2) Mathematical Model: In our approach, a mixed-integer linear programming model is used to formulate the task allocation problem. The objective of the formulation (Eq. (1)) is to maximize the overall reliability of the system. Based on the above transformation, the summation of the logarithmic form of the reliability values of each candidate task equals the product of the reliability values of each candidate task. Let $I$ be the set of the candidate tasks of the R-EHCG and $J$ be the set of the computational units. Hence, we have:

$$
\max \left(\sum_{i \in I} \sum_{j \in J} \log _{2}\left(R_{i j}\right) x_{i j}\right)
$$

s.t. Equation (2) indicates that each $T_{i}$ is allocated only once.

$$
\sum_{j \in J} x_{i j}=1, \forall i
$$

In Eq. (3), we ensure that the correct number of outgoing links $j \rightarrow k$ from $T_{i}$ is enabled, that is, we ensure that our framework will select the corresponding outgoing edges from each candidate task (a task node in the R-EHCG), according to the original TFG.

$$
\sum_{j \in J} \sum_{k \in J} x_{i j k}=n d(i), \forall i
$$

, where $n d(i)$ is the number of descendants of $T_{i}$.

We furthermore define the flow balancing constraint in Eq. (4), which ensures that the path between the candidate tasks is connected, that is, we ensure that when a specific candidate task is selected, then its respective links (edges in the graph) will be selected as well. 


$$
\sum_{k:(i j \rightarrow k)} x_{i j k}-\sum_{k:(k \rightarrow i j)} x_{k i j}=(n d(i)-1)-n a(i)
$$

, where $n a(i)$ is the number of ancestors of $T_{i}$.

Equations (5) to (7) encode the available resources. More specifically, at any given moment, the total main memory and total secondary memory usage, together with the total energy consumption, should not exceed the specified by the designer energy and resource budgets concerning each one of the computational units. We finally integrate the latency constraint in Eq. (8), that is, the overall latency of the system cannot exceed the designer-defined latency threshold. It should be stressed out that for Equation (5)/Equation (8), the first summation considers the computation energy/latency while the second summation considers the communication energy/latency.

$$
\begin{gathered}
\sum_{i \in I} E_{i j} x_{i j}+\sum_{i \in I} \sum_{k \in J} C_{E_{i j k}} x_{i j k} \leq E_{j}, \forall j \\
\sum_{i \in I} M_{i j} x_{i j} \leq M_{j}, \forall j \\
\sum_{i \in I} S_{i j} x_{i j} \leq S_{j}, \forall j \\
\sum_{i \in I} \sum_{j \in J} L_{i j} x_{i j}+\sum_{i \in I} \sum_{j \in J} \sum_{k \in J} C_{L_{i j k}} x_{i j k} \leq L_{\text {thres }}
\end{gathered}
$$

The R-EHCG encodes the case in which a specific task cannot be executed on a specific computational unit, therefore, no extra constraint definition is needed. Moreover, the proposed framework can identify whether a constraint can not be satisfied and report back that no solution can be extracted.

\section{Experimental Evaluation AND Results}

\section{A. Case Study: Aerial Power Line Inspection by a UAV}

We evaluate our proposed framework using a case study based on a real-life problem that involves a UAV-based aerial visual inspection of power transmission lines and towers. Our framework enables the designer to choose, if feasible, the most cost-effective amongst many re-execution approaches according to the application's requirements and constraints. Hence, this problem is ideal to illustrate the capabilities of our framework as it involves a decision support system with real-time performance constraints, as well as various communication and computation constraints involving energy and memory footprints. This application is divided into two main sub-processes. The first subprocess involves the detection of the transmission towers, based on a convolutional neural network. The second sub-process deals with visually inspecting the power transmission lines and detecting obstacles, while also using the transmission lines for navigation purposes. Our case study involves a total of 15 tasks, with varying degrees of computation and communication loads while two of them need to run on a specific device (i.e. the task which deals with the image acquisition has to run on the UAV (edge device) that contains the camera sensor). The reader is referred to [11] for a more detailed description of the casestudy.

\section{B. Experimental Setup}

To demonstrate the generality of the framework, we used five different devices with different characteristics, three as

\begin{tabular}{ccc||cc}
\hline Config. & Edge Device & Hub Device & $\begin{array}{c}\text { Communication } \\
\text { Channel }\end{array}$ & $\begin{array}{c}\text { Bandwidth } \\
\text { (Mbps) }\end{array}$ \\
A & Jetson TX2 & Mi Notebook Pro & Edge $\rightarrow$ Hub & 35 \\
B & Jetson TX2 & Samsung Tab S2 & Edub $\rightarrow$ Edge & 25 \\
C & Odroid XU4 & Mi Notebook Pro & Hub $\rightarrow$ Cloud & 0.5 \\
D & Odroid XU4 & Samsung Tab S2 & Hub $\rightarrow$ Clou & 1.5 \\
E & Raspberry Pi 3 & Mi Notebook Pro & Cloud $\rightarrow$ Hub & 1.5 \\
F & Raspberry Pi 3 & Samsung Tab S2 & & \\
\hline
\end{tabular}

TABLE I: Different Configurations - Channels' Bandwidth

edge devices and two as hub devices. More specifically, the targeted edge devices (as payload to the UAV) were the Nvidia Jetson TX2 (TX2) platform, the Odroid XU4 (XU4) platform, and a Raspberry Pi3 (RPi3) as the high-end, mid-end and lowend edge devices, respectively. We also used a Mi Notebook Pro (Mi Pro) and a Samsung S2 Tablet (S2) as the highend and low-end hub devices, respectively. For all cases, a server equipped with an Intel Xeon E5-2670 CPU was used as the cloud infrastructure. Using the methodology described in Section III-B, the reliability of the devices we used ranged in the following ascending order: cloud unit, Mi Pro, S2, TX2, XU4, and RPi3. Based on this, we built six different edge-hub-cloud configurations as shown in Table I.

Next, after executing the algorithm on all devices and with the aid of profiling tools, the drone's and the tablet's available battery capacity, as well as, a digital energy monitoring device, we gathered the required information: (i) execution time, (ii) energy consumption and (iii) memory requirements for each task, where all these values were used in our optimization framework. The vulnerability factor of each task is given by the designer and it can be computed using existing fault injection techniques or Architecturally Correct Execution analysis, etc. ([4], [18]). In our experiments, we set $\mathcal{T}_{\mathcal{D} \mathcal{R}}=0.02 / \mathcal{T}_{\mathcal{T} \mathcal{R}}=0.06$ for Dual / Triple Re-execution, which derives an optimal solution for system reliability $R_{s}>99.995$. Tasks with vulnerability factor $<0.02$ are executed once, ranged between 0.02-0.06 twice (medium criticality, i.e. image filtering), while $>0.06$ thrice (high criticality, i.e. neural network detection process). However, the designer has the freedom to adjust these thresholds depending on the reliability demands of the application. Moreover, given the nature of our application (typically deployed in rural or rugged areas that have a significant impact on the connectivity), we varied the bandwidths of the four communication channels as shown in Table I.

The optimization algorithm was run on an Intel $\AA$ Core i74720HQ ${ }^{\mathrm{TM}} \mathrm{CPU} @ 2.6 \mathrm{GHz}$ (Memory: 15.9 GB of RAM), using the Gurobi Optimizer 8.1 [19]. For this specific case-study, our framework returned a solution between $\sim 90 \mathrm{~ms}$ for the scenarios where no re-execution was required and $\sim 4 \mathrm{~min}$ for the most time-consuming scenarios where full triple re-execution was used.

\section{Results and Discussion}

To conduct our experiments, we first optimized for performance to find out the allocation that returns minimum latency and how much each reliability mechanism affects the performance of the system. Then we optimized with respect to reliability to detect if any fluctuations in the overall system's reliability occurred, and if so, what is the overall overhead. Even though there are various reliability-aware task allocation works in the literature, this is to our knowledge the first formulation that 


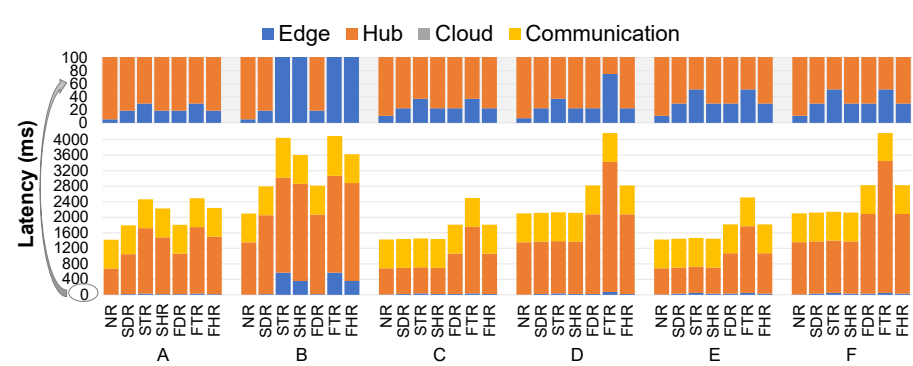

(a) Latency Values when Optimized with respect to Performance

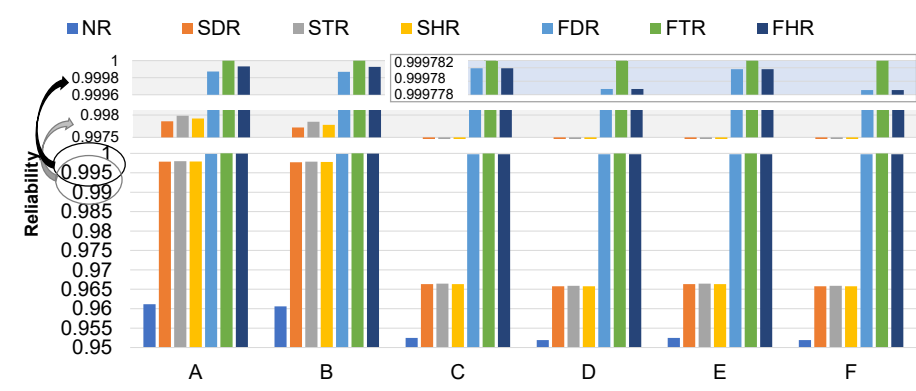

(b) Reliability Values when Optimized with respect to Performance

Fig. 2: Representation of latency and reliability values when optimized with respect to performance

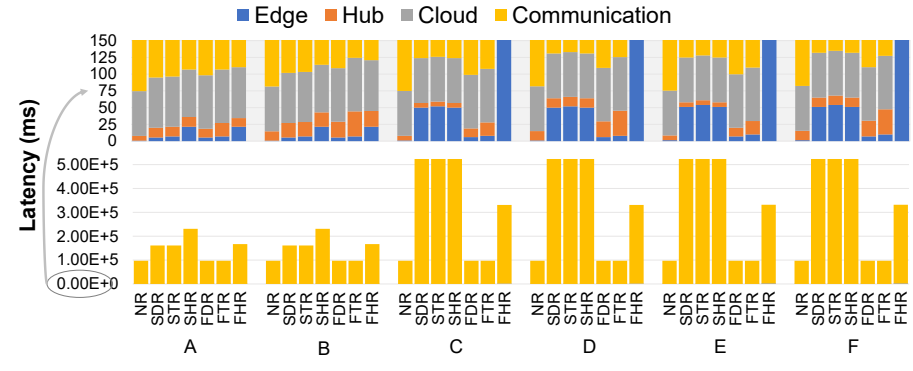

(a) Latency Values when Optimized with respect to Reliability

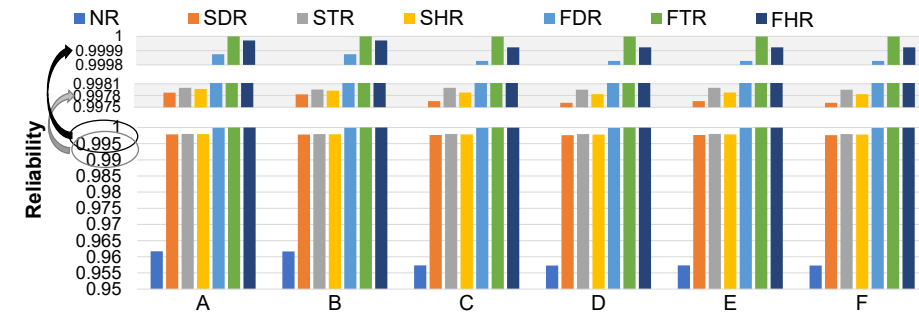

(b) Reliability Values when Optimized with respect to Reliability

Fig. 3: Representation of latency and reliability values when optimized with respect to reliability

optimizes reliability for the specific edge-hub-cloud architecture, hence, any comparison with existing approaches does not apply.

Figures 2a and 3a illustrate the overall latency of the system, that is, the overall computation latency at the edge, hub, and cloud, as well as, the overall communication latency for all communication channels (edge $\rightarrow$ hub, hub $\rightarrow$ cloud, and vice-versa) that took place during the program execution for a specific task allocation. More specifically, Fig. 2a shows the latency values for the case in which the optimization criterion was the maximum performance of the system in terms of latency, while Fig. 3a shows the latency values for the case in which the optimization criterion was maximum reliability. Figures $2 b$ and $3 \mathrm{~b}$ show the reliability of the system in the cases where the optimization criterion was the maximum performance and the maximum reliability, respectively. To better illustrate our results, we present on the top of each main figure zoomed-in snapshots of the results.

For each configuration described in Section IV-B, we consider seven different scenarios as shown in Table II. For each scenario, our framework returns the optimal allocation solution. For example, for NR all tasks will be executed only once, while for STR selected tasks will be executed three times and the rest just once. The rest of the allocation schemes are shown in Table II. Moreover, to show the potency and flexibility of our framework we applied different constraints for the overall latency of the system and the available main memory on some of the computational devices.

In Fig. 2a, in particular, we observe how the latency varies based on the desired reliability level. When maximum performance becomes the primary requirement, we observe that the best performance without any re-execution (NR) occurs in Conf. $A$. This is expected, since the high-end edge and hub devices, respectively, were used. Respectively, the worst performance

\begin{tabular}{cc||ccc}
\hline \multirow{2}{*}{ Scenario \# } & \multirow{2}{*}{ Description } & \multicolumn{3}{|c}{ Modes of Execution } \\
\cline { 3 - 5 } & Single & Dual & Triple \\
1 & No Re-execution (NR) & + & & \\
2 & Selective DR (SDR) & + & + & \\
3 & Selective TR (STR) & + & & + \\
4 & Selective HR (SHR) & + & + & + \\
5 & Full DR (FDR) & & + & \\
6 & Full TR (FTR) & & & + \\
7 & Full HR (FHR) & & + & + \\
\hline
\end{tabular}

TABLE II: Reliability Scenarios

was observed in Conf. $F$ for NR, using low-end edge and hub devices. There are, however, cases in which the allocation cannot be derived straightforwardly by the characteristics of the devices used. In particular, although the combination of devices used in each configuration differs in terms of computation capabilities, the pre-defined tolerable vulnerability threshold plays a decisive role in the resulting task allocation. For example, although in the scenario SDR, Conf. A was expected to get better performance than Conf. $C$, we see that was not the case. Based on the predefined $\mathcal{T}_{\mathcal{D R}}$ threshold for the Conf. $A$ in SDR, $20 \%$ of the tasks were executed only once, while the rest twice. In contrast, for Conf. $C$, in the same scenario, $87 \%$ of the tasks were executed only once.

For all configurations, for scenarios NR, FDR and FTR the tasks were allocated, as it was expected, on the cloud, when maximum reliability was targeted (Fig. 3a), except specific tasks that were restricted to run on the edge and hub devices, respectively. For the rest of the scenarios, although most of the tasks were allocated on the cloud, several tasks were allocated on the edge and hub devices. This, again was due to the predefined tolerable vulnerability threshold. For all scenarios in all configurations, the communication latency was by far greater than the computation latency when optimizing for reliability. This was expected since our case study has to do with realtime high-definition video streaming and the bandwidth of the 


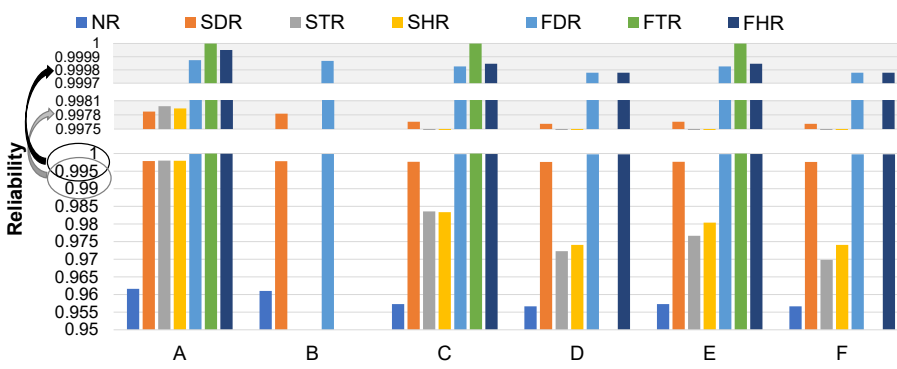

(a) Reliability Values - Latency Constraint $3 \mathrm{sec}$ (same as with and without $50 \%$ memory reduction)

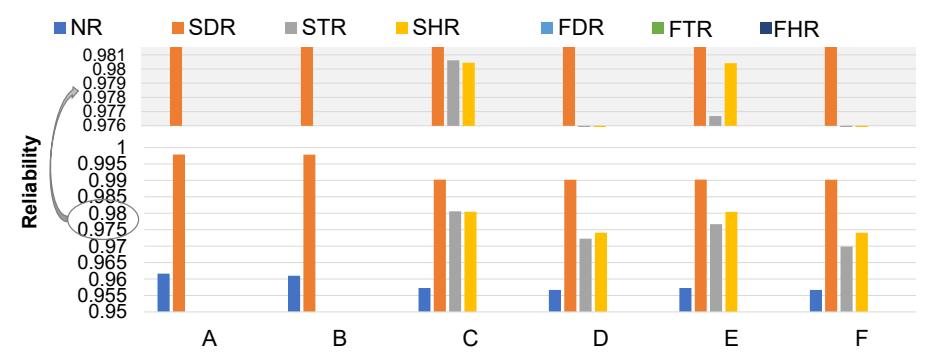

(b) Reliability Values - Latency Constraint 3sec and Memory Capacity Reduced by $80 \%$

Fig. 4: Reliability values subject to performance and memory constraints

communication channels Hub $\rightarrow$ Cloud and Cloud $\rightarrow$ Hub is too small. Compared with Fig. 2a, we observe that there is a considerable increase in the overall latency due to the additional communication latency incurred by shifting most tasks to the cloud, which enforces the need for shifting tasks towards the edge. This happens, as latency is unrestricted in this experiment, but it is constrained in the experiment reported in Fig. 4.

In Figures $2 b$ and $3 b$ we observe that when NR was applied, Conf. A returned the highest reliability while the lowest reliability was obtained in Conf. $F$. Of course, the application is what drives the desired reliability levels and thus the framework enables the designer to extract them. An interesting observation in Figure 2b, for Conf. $C$, is that the reliability of the scenarios FDR and FHR is the same. Although this seems odd, the given vulnerability threshold is what causes this, since the vulnerability factor of each candidate task differs. More specifically, the vulnerability factor of each candidate task and the designer-specified tolerable vulnerability threshold define the transformation of the EHC graph to its R-EHC version, which in the end, along with the constraints will finally select the most suitable allocation. Within this reasoning, the framework returns the same allocation for both FDR and FHR cases. The same applies to Confs. $D, E$, and $F$.

As shown in Figure 4a, when the overall latency of the system was constrained to be less than $3 \mathrm{sec}$, there no solution was feasible for the scenario where TR was applied in Confs. B, D, and $F$. It should be noted that similar results were obtained when we reduced the available main memory capacity by $50 \%$ for both edge and hub devices. However, if we reduce the available memory capacity by $80 \%$, we observe that no solution exists for both DR and TR for all configurations (Fig. 4b). Within this context, our framework enables the designer to evaluate trade-offs between reliability, performance, and energy (within the application's constraints) and also experiment with different device-driven parameters (i.e. computational latency, memory constraints, etc.) to clearly identify the optimal approach for maximizing reliability while at the same time, satisfying strict constraints.

\section{CONClusion \& Future Work}

We propose a framework that is able to find the optimal costeffective allocation of an application's tasks among the edgehub-cloud computing paradigm, in such a way, that the overall reliability of the system is maximized. Our framework considers various resource constraints, as well as, the overall latency of the system. Furthermore, it takes into consideration the designer-defined tolerable thresholds which can result in various allocation schemes such as selective and full dual and triple re-execution, as well as, selective and full hybrid approaches. This is crucial because our framework allows the designer to get inside information about the system by determining which devices and configurations are suitable for the targeted application under some specific constraints. For the future, we aim to extend the proposed framework to architectures with multiple edge and/or hub devices, support multiple objectives such as the optimal trade-off between the latency and reliability of the system, as well as non-ideal communication channel operation.

\section{REFERENCES}

[1] C. Chang et al., "Internet of things (iot) and new computing paradigms," Fog and Edge Computing: Principles and Paradigms, pp. 1-23, 2019.

[2] M. Ottavi et al., "Dependable multicore architectures at nanoscale: The view from europe," IEEE Design \& Test, vol. 32, no. 2, pp. 17-28, 2014.

[3] V. Fratin et al., "Code-dependent and architecture-dependent reliability behaviors," in 48th DSN. IEEE, 2018, pp. 13-26.

[4] S. S. Mukherjee et al., "A systematic methodology to compute the architectural vulnerability factors for a high-performance microprocessor," in 36th MICRO, Dec 2003, pp. 29-40.

[5] M. Chiang and T. Zhang, "Fog and iot: An overview of research opportunities," IEEE IoT Journal, vol. 3, no. 6, pp. 854-864, 2016.

[6] A. K. Singh et al., "Mapping on multi/many-core systems: Survey of current and emerging trends," in 50th DAC, May 2013, pp. 1-10.

[7] J. Zhou et al., "Variation-aware task allocation and scheduling for improving reliability of real-time mpsocs," in DATE. IEEE, 2018, pp. 171-176.

[8] K. Kolomvatsos and C. Anagnostopoulos, "Multi-criteria optimal task allocation at the edge," Future Generation Computer Systems, vol. 93, pp. $358-372,2019$.

[9] V. B. C. d. Souza et al., "Handling service allocation in combined fogcloud scenarios," in ICC. IEEE, 2016, pp. 1-5.

[10] H. Shah-Mansouri and V. W. S. Wong, "Hierarchical fog-cloud computing for iot systems: A computation offloading game," IEEE IoT Journal, vol. 5, no. 4, pp. 3246-3257, Aug 2018.

[11] A. Kouloumpris et al., "Metis: Optimal task allocation framework for the edge/hub/cloud paradigm," in COINS. ACM, 2019, pp. 128-133.

[12] X. Hou et al., "Fog based computation offloading for swarm of drones," in ICC. IEEE, 2019, pp. 1-7.

[13] L. Dong et al., "Reliability-aware offloading and allocation in multilevel edge computing system," IEEE Trans. on Reliability, pp. 1-12, 2019.

[14] C. Liu et al., "Latency and reliability-aware task offloading and resource allocation for mobile edge computing," in IEEE Globecom Workshops (GC Wkshps), Dec 2017, pp. 1-7.

[15] A. Kouloumpris et al., "Reliability-aware task allocation latency optimization in edge computing," in 25th IOLTS, July 2019, pp. 200-203.

[16] H. Zhang et al., "Resource budgeting for reliability in reconfigurable architectures," in 53nd DAC, June 2016, pp. 1-6.

[17] A. Wang et al., "Xpro: A cross-end processing architecture for data analytics in wearables," in 44th ISCA. NY, USA: ACM, 2017, pp. 69-80.

[18] V. Sridharan and D. R. Kaeli, "Using hardware vulnerability factors to enhance avf analysis," in ACM SIGARCH Computer Architecture News, vol. 38, no. 3. ACM, 2010, pp. 461-472.

[19] L. Gurobi Optimization, "Gurobi optimizer reference manual," 2018. 